\title{
Dynamical properties of discrete breathers in curved chains with first and second neighbor interactions
}

\author{
M. Ibañes, ${ }^{1}$ J. M. Sancho, ${ }^{1}$ and G. P. Tsironis ${ }^{1,2, *}$ \\ ${ }^{1}$ Department d'Estructura i Constituents de la Matèria, Universitat de Barcelona, Diagonal 647, E-08028 Barcelona, Spain \\ ${ }^{2}$ Center for Nonlinear Studies, MS-B258, Los Alamos National Laboratory, Los Alamos, New Mexico 87545 \\ (Received 16 November 2000; revised manuscript received 2 October 2001; published 15 March 2002)
}

\begin{abstract}
We present the study of discrete breather dynamics in curved polymerlike chains consisting of masses connected via nonlinear springs. The polymer chains are one dimensional but not rectilinear and their motion takes place on a plane. After constructing breathers following numerically accurate procedures, we launch them in the chains and investigate properties of their propagation dynamics. We find that breather motion is strongly affected by the presence of curved regions of polymers, while the breathers themselves show a very strong resilience and remarkable stability in the presence of geometrical changes. For chains with strong angular rigidity we find that breathers either pass through bent regions or get reflected while retaining their frequency. Their motion is practically lossless and seems to be determined through local energy conservation. For less rigid chains modeled via second neighbor interactions, we find similarly that chain geometry typically does not destroy the localized breather states but, contrary to the angularly rigid chains, it induces some small but constant energy loss. Furthermore, we find that a curved segment acts as an active gate reflecting or refracting the incident breather and transforming its velocity to a value that depends on the discrete breathers frequency. We analyze the physical reasoning behind these seemingly general breather properties.
\end{abstract}

DOI: 10.1103/PhysRevE.65.041902

PACS number(s): 87.15.-v, 63.20.Pw, 36.20.-r

\section{INTRODUCTION}

The basic question to be addressed in this paper relates to the dynamics of space localized lattice oscillation modes referred to as intrinsic localized modes (ILM's) or discrete breathers (DB's) in elastic polymeric chains with rigidity [1-20]. Unlike solitons, DB's appear to be generic modes of nonlinear lattices provided the latter are equipped with these two basic ingredients: nonlinearity in the interactions and lattice discreteness. During the last several years there has been an abundance of theoretical work dealing with various aspects of DB properties, including generation [1-8], rigorous existence [6], dynamics and mobility [9], thermodynamics and statistical properties [11,13], quantum features $[10,16]$, etc. As a result, many of the basic DB properties have been revealed and are now relatively well understood. On the experimental front, a recent avalanche of results in a large variety of systems demonstrating DB presence, or at least strong indications for it, has set the whole area on very solid and promising new grounds [17-20]. There can now be more precise studies of specific condensed matter, chemical and biological systems, as well as discussion on the design of breather based materials. The work to be presented in this paper points also in this direction, as it attempts to deviate from simple one-dimensional lattice models by introducing one additional new element, that of single chain elasticity. We will thus be concerned here with polymeric chains of masses coupled with springs that can move, in principle, in the whole $(x, y)$ plane and are characterized by local and

\footnotetext{
*Permanent address: Department of Physics, University of Crete and Foundation for Research and Technology-Hellas, P. O. Box 2208, 71003 Heraklion, Crete, Greece.
}

global elastic properties. Our basic question is how energy localization in the form of DB's interplays with single chain polymer elasticity.

Our basic model will be an arbitrarily shaped chain of equal masses coupled typically via nonlinear springs involving only two-body polynomial-type interactions. Unlike recent work on the same general topic [21], we will not consider long-range interactions presently so that we keep the complexity of the model and the number of parameters used to a minimum. We will instead use first and second neighbor interactions that have the same form but enter with different strengths and equilibrium distances. After the first stage of our analysis we will initially "turn off" the second neighbor interaction and study the simplest possible nonlinear bead and spring model, while subsequently the second neighbor interaction will be included and comparisons will be made between the two cases. Since our main interest is in understanding the physics of breathers in biomolecules such as proteins [22] rather than general homopolymers, we will have to somehow restrict our study to rigid and quasirigid polymer geometries. This can only be done artificially through constraints when only first neighbor interactions are taken into account due to the high level of degeneracy of the chain. The source of the latter is geometric since there are multiple equilibrium states that are distributed in various configurations on the plane while preserving the nearest neighbor equilibrium distances. However, when the second neighbor interaction is turned on, the chain degeneracy reduces substantially and there is no need for additional external constraints.

As mentioned earlier, the questions to be addressed here will focus on the interplay of energy localization in the form of DB's and biopolymer spatial structure. Since this presents a rather broad topic we will narrow the questions in this study down to the following three: (a) can a stable breather 
be generated in a curved polymer with some rigidity; (b) can a breather propagate in curved portions of a polymer and what are the features of its kinetics as it traverses straight and curved segments of the polymer; and (c) can the breather motion affect the polymer geometry? In order to tackle these questions it is critical to have a systematic method of generation of localized DB modes with different frequencies and also to be able to make them mobile at desired possible velocities. These systematic tools already exist and with appropriate modifications and improvements have been used in the present study. For the numerically exact generation of one-dimensional DB's we used the construction from the anticontinuous limit [8], while for a faster, yet accurate approximate construction we used the algebraic method [23]. Furthermore, for rendering the DB mobile we used a simple variant of the pinning mode excitation method [9]. These methods provide systematic tools for breather construction and dynamics.

The basic results of this study can be summarized in the following: (a) Discrete breathers can be generated in curvilinear polymeric chains, are surprisingly stable, and can propagate quite easily through curved parts of the chain. They can be reflected at interfaces of regions with different curvature without losing their integrity. (b) The evolution of DB's seems to be taking place through conservation of their internal and translational energy. This law of energy conservation enables appropriate internal to/from translational energy transformations that, in turn, dictate the DB shape and extent as well as kinetics while the DB frequency does not change. Some small constant energy loss is observed in the less rigid chains that forces breathers to change slightly their form while propagating. (c) The presence of a DB in an flexible polymeric chain induces large chain amplitude motion that destabilizes the polymer when only first neighbor interactions are used, but without much affecting the breather if second neighbor interactions are incorporated. The specificity of this effect depends primarily on the breather frequency or internal energy. Based on these results, which will be elaborated on below, we can make some more general statements regarding the possible role of breathers in biopolymers. It appears that stable breather modes cannot only be generated easily in curved chains but can also traverse parts of the polymer in an adaptive fashion retaining their basic features. They can thus transfer energy packets across segments of the polymer. These features make breathers very appealing energy managers of internal biopolymer organization since they accomplish three functions simultaneously, viz., they can be generated locally through chemical to localized vibrational energy conversion, facilitate energy transfer through adaptive transport, and possibly convert localized vibrational to mechanical energy at an alternative site.

In order to cover the topics briefly touched above we will use the following structure in the remainder of the paper. In Sec. II, we introduce the model and discuss the aspects of interest by making a connection with biomolecular models. Since we are mostly interested in polymers with some rigidity, we introduce in Sec. III a first neighbor model with constraints that simulate the latter. The model rigidity is set by

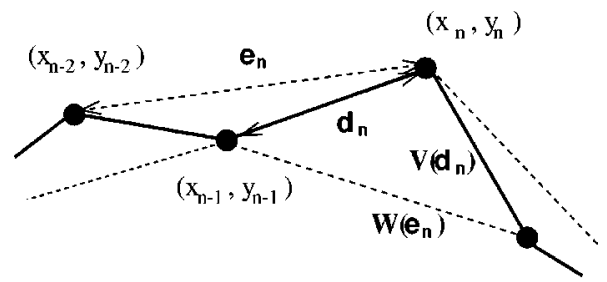

(a)

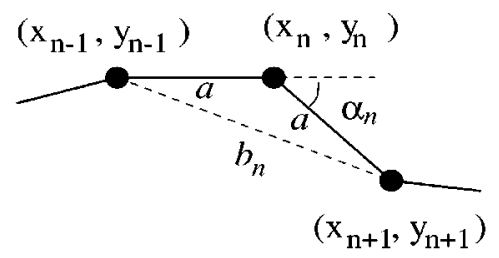

(b)

FIG. 1. (a) Picture of the model. The interaction between nearest neighbors is controlled by potential $V\left(d_{n}\right)$ and depends on their relative distance $d_{n}$ (full lines). There is also an interaction between second neighbors controlled by $W\left(e_{n}\right)$, which depends on their relative distance $e_{n}$ (broken lines). (b) Equilibrium distances $a_{n}=a$ and $b_{n}$ between adjacent masses and next nearest neighbors, respectively, as a function of the relative angle $\alpha_{n}$.

constraining the angles between adjacent polymeric segments to constant but arbitrary values. This reduced model is the workhorse of our study since it gives the basic features of breather dynamics in curved spaces. In this section, we will give all details regarding generation of mobile DB's and we will present the results of the DB dynamics in curves. We will also demonstrate here one of the basic results of this work, viz., that of the adaptive breather evolution governed by a local form of the law of conservation of energy. We will finally investigate the dependence of the phenomenon on the DB frequency. In this section, we will also test the stability of our results in chains that are weakly perturbed with static disorder. In Sec. IV, we will include the second neighbor interaction and we will repeat some of the previous work but in the fully two-dimensional model. The presence of this second neighbor interaction augments the structural stability of the chain and assists in the DB stability. Finally, in Sec. V, we will conclude by presenting a summary of the work and an outlook on this work for its potential use in biomolecules.

\section{THE MODEL}

Let us consider a curvilinear polymer chain such as the one depicted in Fig. 1(a). The chain consists of $N$ molecular units interacting through pairwise two-body interactions. We will make the following three assumptions: (a) all unit masses are identical and equal to $m$, (b) there are only first and second neighbor interactions between the molecular units, and (c) the polymer lies on the $(x, y)$ plane. Clearly in a true biomolecular chain none of these assumptions is strictly valid. They are made here, however, since they are compatible with the basic spirit of this work, viz., keeping model complexity to a minimum so that the role of nonlinear dynamical localization becomes transparent. If the polymer 
we have in mind is a protein segment, assumption (a) would be compatible with considering the units as being part of a backbone consisting of carbon or nitrogen atoms that only differ slightly and ignoring amino acid side chains, (b) with assuming very quickly decaying intermolecular interactions as well as the absence of hydrogen bonding, and (c) the absence of $\alpha$ helices. While these are reasonable for some protein segments, they are not true in general; they can easily be lifted, however, in a more specific system study.

Each mass unit in the chain is labeled by an index $n$, while its location is specified through the pair $\left(x_{n}, y_{n}\right)$ denoting its location on the plane with respect to an absolute Cartesian system. Since we will use first and second neighbor interaction potentials we need to introduce the following two Euclidean distances:

$$
\begin{aligned}
& d_{n}=\left[\left(x_{n}-x_{n-1}\right)^{2}+\left(y_{n}-y_{n-1}\right)^{2}\right]^{1 / 2}, \\
& e_{n}=\left[\left(x_{n}-x_{n-2}\right)^{2}+\left(y_{n}-y_{n-2}\right)^{2}\right]^{1 / 2} .
\end{aligned}
$$

We note that $d_{n}, e_{n}$ are simply the distances on the plane between the $n$th unit and the $(n-1)$ th and $(n-2)$ th units, respectively. The polymer chain plasticity as well as rigidity are controlled by the ensemble of first and second neighbor constant equilibrium distances $\left\{a_{n}\right\}$ and $\left\{b_{n}\right\}$, respectively. The constant $a_{n}$ is the equilibrium oscillator distance between units $n$ and $(n-1)$, while $b_{n}$ is that between the $n$th and $(n-2)$ th units. The explicit configuration of these two sets of constants fixes the desired equilibrium geometry of the polymer chain. Although the derivation presented here is general, in the following sections we will restrict ourselves to the case $a_{n}=a \forall n$, and $b_{n}$ depends on the geometrical structure we want to study, i.e., it fixes the relative angles $\alpha_{n}$ [see Fig. 1(b)].

Let us finally define the interaction potentials. Although we will present below general equations of motion for arbitrary pairwise potentials, in the specifics we will use the following Fermi-Pasta-Ulam (FPU) -type potentials:

$$
\begin{aligned}
& V\left(d_{n}\right)=K_{1} \frac{\left(d_{n}-a_{n}\right)^{2}}{2}+\beta_{1} \frac{\left(d_{n}-a_{n}\right)^{4}}{4}, \\
& W\left(e_{n}\right)=K_{2} \frac{\left(e_{n}-b_{n}\right)^{2}}{2}+\beta_{2} \frac{\left(e_{n}-b_{n}\right)^{4}}{4} .
\end{aligned}
$$

The Hamiltonian for the planar polymer chain can be then written as

$$
H=\sum_{n} \frac{m}{2}\left(\dot{x}_{n}^{2}+\dot{y}_{n}^{2}\right)+\sum_{n}\left\{V\left(d_{n}\right)+W\left(e_{n}\right)\right\},
$$

where the index $n$ runs over all polymer masses. The resulting equations of motion are

$$
m \ddot{x}_{n}=-\frac{\partial}{\partial x_{n}} U_{n}, \quad m \ddot{y}_{n}=-\frac{\partial}{\partial y_{n}} U_{n}
$$

with

$$
U_{n}=V\left(d_{n}\right)+V\left(d_{n+1}\right)+W\left(e_{n}\right)+W\left(e_{n+2}\right),
$$

or, more explicitly,

$$
\begin{aligned}
m \ddot{x}_{n}= & \frac{x_{n}-x_{n-1}}{d_{n}} f_{n}-\frac{x_{n+1}-x_{n}}{d_{n+1}} f_{n+1}+\frac{x_{n}-x_{n-2}}{e_{n}} g_{n} \\
& -\frac{x_{n+2}-x_{n}}{e_{n+2}} g_{n+2}, \\
m \ddot{y}_{n}= & \frac{y_{n}-y_{n-1}}{d_{n}} f_{n}-\frac{y_{n+1}-y_{n}}{d_{n+1}} f_{n+1}+\frac{y_{n}-y_{n-2}}{e_{n}} g_{n} \\
& -\frac{y_{n+2}-y_{n}}{e_{n+2}} g_{n+2},
\end{aligned}
$$

where

$$
f_{n} \equiv-d V\left(d_{n}\right) / d d_{n}, \quad g_{n} \equiv-d W\left(e_{n}\right) / d e_{n} .
$$

The form of the equations suggests the introduction of the relative variables $\xi_{n}$ and $\rho_{n}$ that will be also normalized using a characteristic length $a$,

$$
\begin{gathered}
\xi_{n} \equiv\left(x_{n}-x_{n-1}\right) / a, \\
\rho_{n} \equiv\left(y_{n}-y_{n-1}\right) / a .
\end{gathered}
$$

In terms of these new variables the Euclidean distances become $d_{n}=a^{2}\left[\xi_{n}^{2}+\rho_{n}^{2}\right]^{1 / 2}$ and $e_{n}=a^{2}\left[\left(\xi_{n}+\xi_{n-1}\right)^{2}+\left(\rho_{n}\right.\right.$ $\left.\left.+\rho_{n-1}\right)^{2}\right]^{1 / 2}$, respectively. Introducing now the adimensional complex coordinate $z_{n}=\xi_{n}+i \rho_{n}$, we obtain the following compact form for the equations of motion:

$$
\begin{aligned}
\ddot{z}_{n}= & R_{n+1}+R_{n-1}-2 R_{n} \\
& +Q_{n+2}-Q_{n-1}-Q_{n}+Q_{n-1}
\end{aligned}
$$

with

$$
\begin{gathered}
R_{n}=-\frac{z_{n}}{\left|z_{n}\right|} f_{n}, \\
Q_{n}=-\frac{z_{n}+z_{n-1}}{\left|z_{n}+z_{n-1}\right|} g_{n},
\end{gathered}
$$

where for the specific FPU potentials of Eqs. (3) and (4), we have

$$
\begin{gathered}
R_{n}=\frac{z_{n}}{\left|z_{n}\right|}\left[\left(\left|z_{n}\right|-\tilde{a}_{n}\right)+\gamma_{1}\left(\left|z_{n}\right|-\tilde{a}_{n}\right)^{3}\right], \\
Q_{n}=\frac{z_{n}+z_{n-1}}{\left|z_{n}+z_{n-1}\right|}\left[\lambda\left(\left|z_{n}+z_{n-1}\right|-\tilde{b}_{n}\right)\right. \\
\left.\quad+\gamma_{2}\left(\left|z_{n}+z_{n-1}\right|-\tilde{b}_{n}\right)^{3}\right],
\end{gathered}
$$

where time has been adimensionalized as $t \rightarrow t \sqrt{K_{1} / m}$ and the parameters are $\gamma_{1}=a^{2} \beta_{1} / K_{1}, \quad \lambda=K_{2} / K_{1}, \quad \gamma_{2}$ $=a^{2} \beta_{2} / K_{1}, \tilde{a}_{n}=a_{n} / a$, and $\tilde{b}_{n}=b_{n} / a$.

Equations (8) and (9) or the compact forms in complex variables (12) are the basic equations to be used in this work. 
A Runge-Kutta algorithm of fourth order has been used to integrate the equations of motion with a time step $\delta t$ $=0.005$.

\section{A RESTRICTED MODEL}

\section{A. A modified FPU equation}

In the preceding section, we wrote the general equations of motion that govern an arbitrary polymeric chain on the plane when the masses interact with specific first and second neighbor interactions. If only first neighbor interactions are taken into account the resulting chain is characterized by a high degree of flexibility, multiplicity of stationary points, and a high degree of degeneracy and instabilities $[24,25]$. While the study of this completely flexible polymeric chain is interesting, it is not in tune with our main objective here- to work with models that are compatible with biopolymers. Since the latter are characterized by a high degree of rigidity, we need to introduce external, additional constraints, if we want to stay at the lowest level of polymer description, viz., that of retaining only first neighbor interactions. To achieve this goal, we introduce rigidity in this model by constraining the relative angles between adjacent bonds to constant but arbitrary values. This can be accomplished easily if we write Eq. (12) with only first neighbor interactions using the polar representation $z_{n}=r_{n} \exp \left(i \theta_{n}\right)$ and introduce additionally the local relative displacement $\tau_{n} \equiv r_{n}-\tilde{a}_{n}$. The constraint of fixed relative angles in these new variables reads $\ddot{\theta}_{n}=\dot{\theta}_{n}=0, \theta_{n}(t)=\theta_{n}(0)$.

We finally obtain a reduced dynamical model described by the equations

$$
\begin{gathered}
\ddot{\tau}_{n}=\epsilon_{n+1, n} \hat{f}_{n+1}+\epsilon_{n, n-1} \hat{f}_{n-1}-2 \hat{f}_{n}, \\
\epsilon_{n, n-1}=\cos \left(\theta_{n}-\theta_{n-1}\right), \\
\hat{f}_{n}=-f_{n} .
\end{gathered}
$$

In the specific case of the FPU potential of Eq. (3), we have

$$
\hat{f}_{n}=\tau_{n}+\gamma_{1} \tau_{n}^{3}
$$

We will term Eqs. (17) and (18) modified Fermi-Pasta-Ulam (mFPU) equation. For a straight line geometry, $\theta_{n}=\theta^{\circ} \forall n$, the mFPU is equivalent to the FPU model $\left(\epsilon_{n+1, n}=1\right)$, which can be obtained from Hamiltonian (5) in a onedimensional space. In contrast, for an arbitrary geometry, there is no Hamiltonian associated with the mFPU equations of motion except in the form of a two-dimensional one with holonomic constraints.

For the numerically exact generation of one-dimensional DB's, we used the construction from the anticontinuous limit [8], or an alternative algebraic method [23] that renders accurate DB's rapidly. In Fig. 2 different breathers are shown for several values of $\epsilon_{n+1, n}=C \leqslant 1 \forall n$, which correspond to circular or zig-zag geometries. Note that exact DB's of the mFPU model with $\epsilon_{n+1, n}=C \forall n$ correspond to intermediate steps of the anticontinuous limit method for generating exact

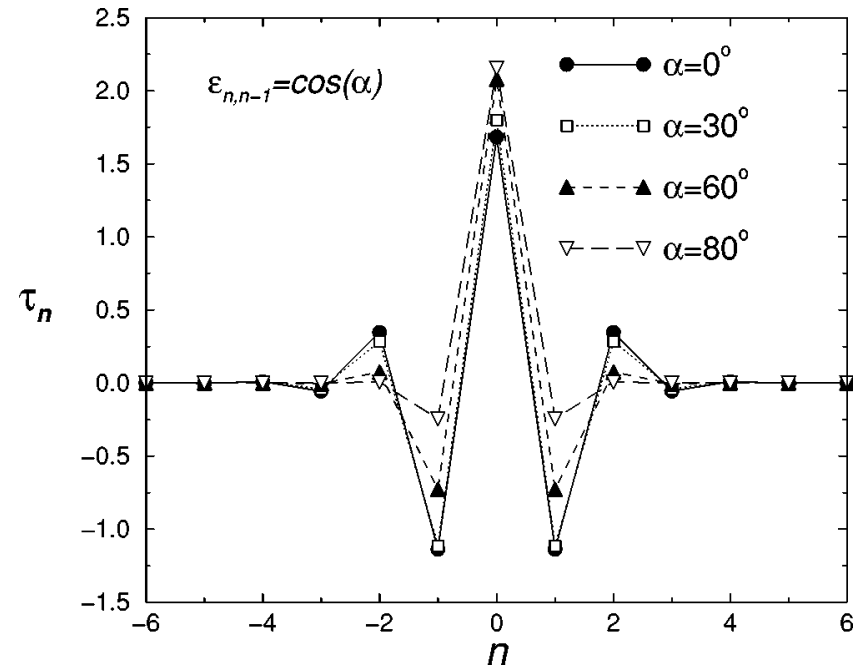

FIG. 2. DB solutions (algebraic method) with $T_{b}=2.122$ for the mFPU model for different values of $\alpha$. As expected, the solutions tend to the one-dimensional DB solution corresponding to $\alpha=0$.

DB's in FPU chains $\left(\epsilon_{n+1, n}\right.$ varies smoothly from 0 to 1 in the anticontinuous limit method).

For rendering DB's mobile, we use a simple modification of the pinning mode method [9]. We assign an initial DB velocity proportional to the gradient of the absolute value of its amplitudes; the velocity vector thus constructed is parallel to the dominant antisymmetric stability eigenvector of Ref. [9]. The resulting mobile DB is not exact and thus sheds initially a phononic wave packet that travels at the sound velocity. With this method and for a sound velocity equal to unity, we are able to render DB's mobile within the velocity window $v \in[0.035,0.15]$, i.e., the fastest DB generated in this fashion has a speed approximately equal to $15 \%$ of the sound velocity.

\section{B. Breather motion in curved rigid polymers}

After having explained the restricted model structure and the methods of construction of mobile DB's, we are now in a position to perform numerical experiments dealing with the dynamics of DB's when passing through a curved region. In particular, we consider a hairpin geometry such as the one depicted in Fig. 3, characterized by angle $\alpha$ and equilibrium distances between particles $a$; the specific choice of the geometry has been inspired by $\beta$ sheets of proteins. Neverthe-

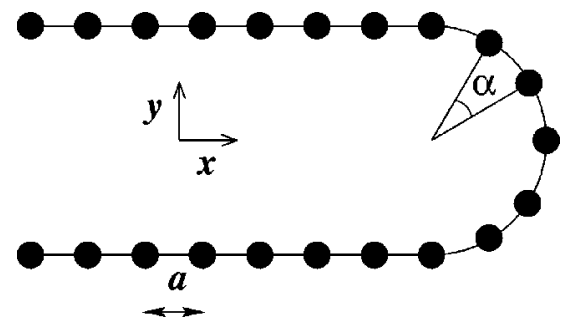

FIG. 3. Hairpin geometry characterized by angle $\alpha$ and equilibrium distance $a$ between adjacent particles. 

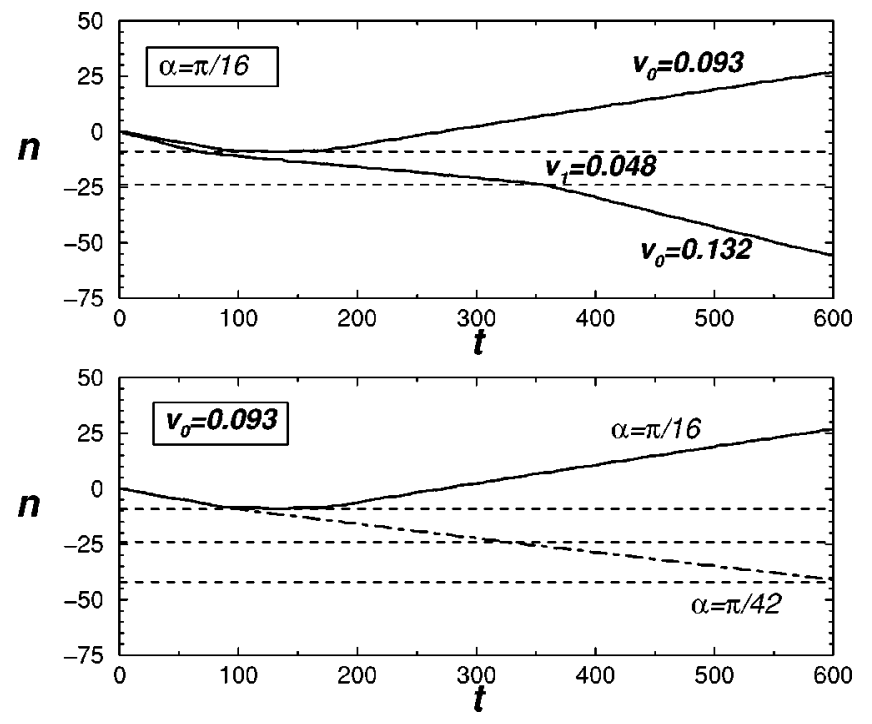

FIG. 4. Site where the center of the DB is located as a function of time. The DB starts moving in the straight region of the hairpin. The curved region is inside the horizontal dashed lines. (Top) For a given curvature, $\alpha=\pi / 16$, the DB enters into the curved region or rebounces depending on the initial velocity. (Bottom) For a given initial velocity, depending on the curvature, the DB enters into the curved region or rebounces. The parameter values are $K_{1}=\beta_{1}=1$ and $a=10$ in this work.

less, the general kinetic breather results are not specific to this geometry, as alternative curves would not affect the results qualitatively.

We first construct an exact FPU breather, corresponding to an exact DB of the mFPU model in a straight chain geometry, with period $T_{b}=2.122$. The parameter values for the FPU potential (3) are $K_{1}=\beta_{1}=1$ and $a_{n}=a=10 \forall n$. The amplitude of the central site of the DB is $1.68 \ll a$. The resulting $\mathrm{DB}$ moves with velocities in the range $v_{\text {min }} \approx 0.03$ and $v_{\text {max }} \approx 0.15$, where length is measured in $a$ units. These velocities are much slower than the sound velocity, $c=1$.

In Fig. 4, we plot the position of the DB as a function of time for different initial velocities (top) and for different hairpin geometries (bottom); we see that a DB traverses a curved region or rebounces depending on its initial velocity and on the local curvature. For a given hairpin geometry, the DB rebounces for small velocities, while for higher velocities it traverses the curved region (top picture). For a given initial velocity in the straight region, the DB rebounces or enters into the curved region depending on $\alpha$. There is a critical curvature below which the DB is able to pass and above which the DB is reflected (bottom picture). In some cases, for critical velocities or critical angles $\alpha$, the DB is trapped in the bend. When rebouncing, the DB velocity remains unchanged. In contrast, when traversing the curved region, the $\mathrm{DB}$ velocity decreases but once the $\mathrm{DB}$ reaches the other straight segment it recovers the initial velocity.

Let us now consider the DB velocities when the DB enters the bend. The velocity inside the curved region for a given hairpin geometry as a function of the initial velocity in the straight segment is plotted in Fig. 5(a). As the initial velocity increases the velocity inside the curved region also
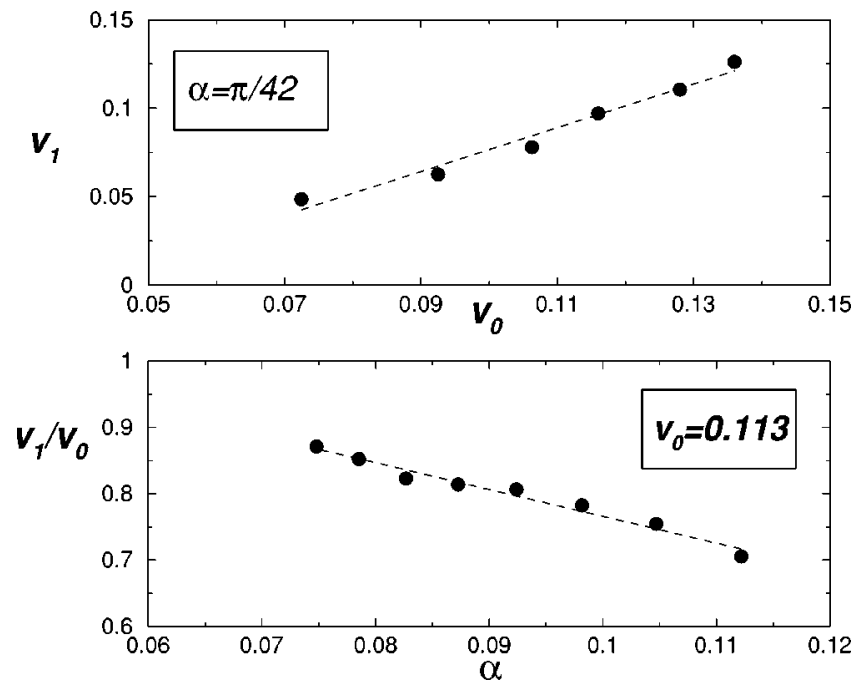

FIG. 5. (Top) Velocity inside the curved region of the hairpin $v_{1}$ as a function of the velocity in the straight region $v_{0}$ for a hairpin with $\alpha=\pi / 42$. (Bottom) Ratio between velocities inside $\left(v_{1}\right)$ and outside $\left(v_{0}\right)$ the hairpin as a function of the characteristic angle of the hairpin. All the data in (b) correspond to $v_{0}=0.113$. Broken lines are a guide to the eye.

increases in a seemingly linear fashion. In Fig. 5(b), we plot for a fixed initial velocity, the velocity inside the curved region as the curvature increases. As the curvature decreases, the reduction of the velocity inside the curved region decreases. Figures 5(a) and 5(b) correspond to initial velocities above the critical one and to hairpin angles below the critical value, respectively.

In Fig. 6, we plot the amplitude of oscillations of different sites of the chain as a function of time when a DB goes into the curved region. We have checked that the DB maintains its amplitude and period while traversing the curved region. The main differences between oscillations of masses outside and inside the curved region is the length of the time interval

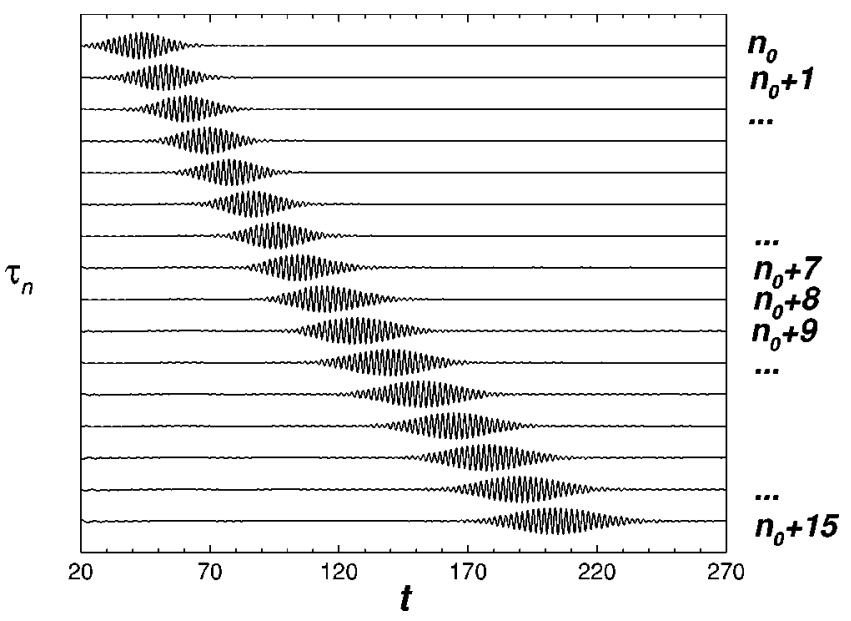

FIG. 6. DB amplitude $\tau_{n}$ as the DB's moves along a straight line and enters into a curved region, which starts at site $n_{0}+8$. As all $\tau_{n}$ oscillate around zero, we have displaced them by $\tau_{n}=\tau_{n}+n C$, where $C=0.4$. The characteristic angle of the hairpin is $\alpha=\pi / 25$. 


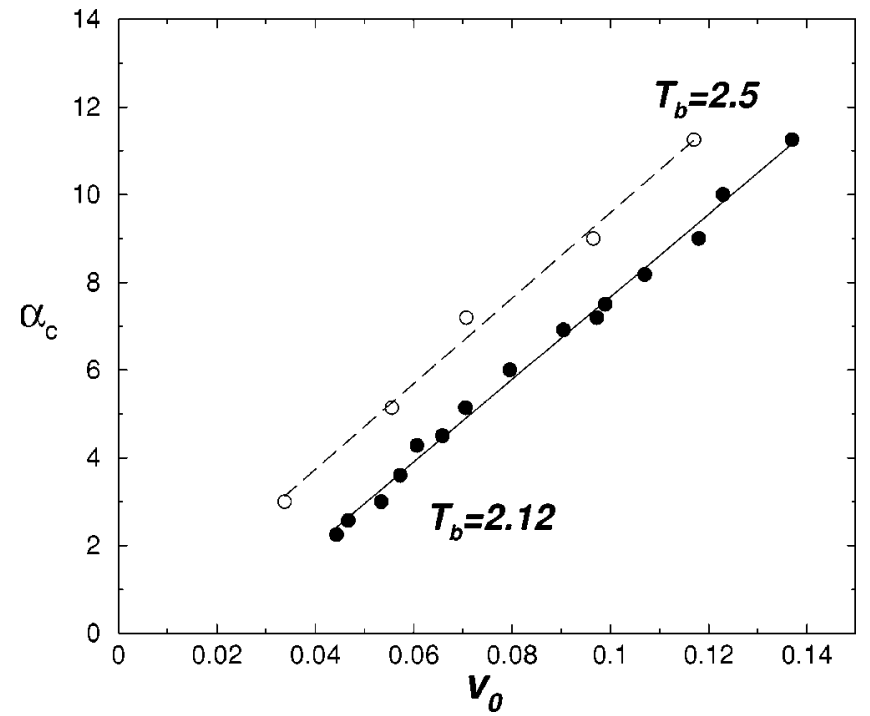

FIG. 7. Critical angle for a DB passing a hairpin structure as a function of the velocity in the straight region, just before entering the curved region. Empty circles correspond to results for a DB with period $T_{b}=2.5$ and full circles to a DB with $T_{b}=2.122$. The angle is given in degrees. The sound velocity is 1 .

during which the mass oscillates. In the curved region, since the velocity is smaller, the time interval is larger.

We have also computed the critical angle for DB's with two different frequencies (see Fig. 7). For the range of velocities we are able to move the DB, the critical angle above which the DB can enter into the bend increases linearly with the incident velocity. As the frequency decreases, the critical angle increases. In other words, for a given initial velocity a DB with lower frequency traverses more easily the curved region than a DB with a higher frequency.

Although the results presented here focus on those curved regions corresponding to hairpin geometries, we also have checked what occurs for different geometries, such as two straight linked segments forming a total angle $\alpha$ and a curved part given by a hyperbolic tangent. The results are qualitatively the same as for a perfect hairpin geometry, the only difference being the specific values of the critical angle and the velocities inside the curved regions. We have also tested this behavior for disordered chain geometries: taking the hairpin geometry as reference, we slightly perturb the relative angles between particles and launch a DB on this imperfect hairpin geometry. If this disorder is small enough the behavior of the DB is qualitatively the same.

\section{Energetics of breather dynamics}

In the preceding section, we addressed the specifics of DB motion in curved geometry for an angle constrained model with only first neighbor interaction. We found that DB's either propagate through the bend or reflect while they principly keep their identity and basic features. We will search in this section the roots of this behavior and see that they are determined quite simply by local DB energy conservation. Let us begin by constructing via the numerically exact procedure from the anticontinuous limit a static, i.e., nonmoving

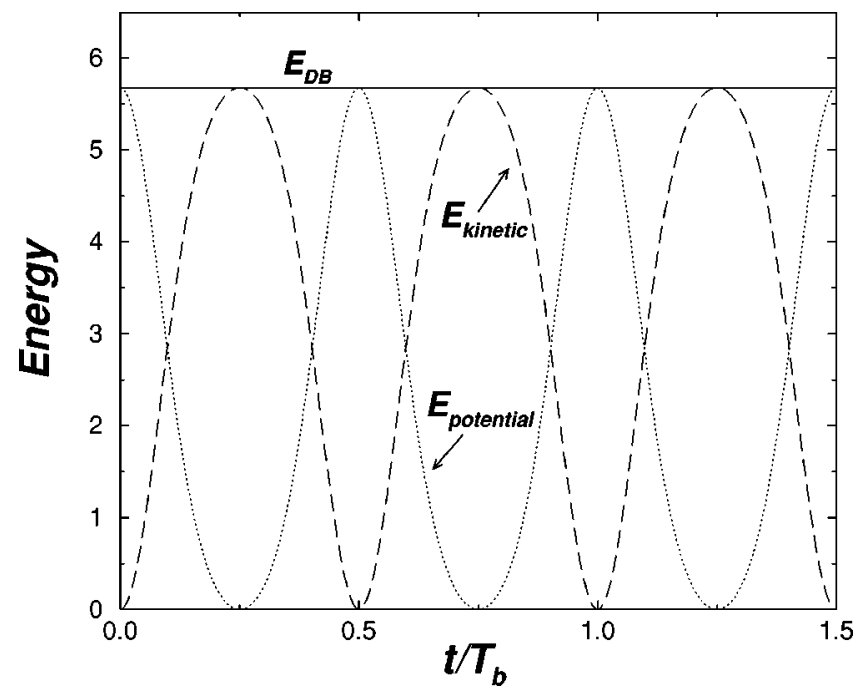

FIG. 8. Energy of a static DB with $T_{b}=2.122$ in a straight line (FPU model). Energy is in units of $a^{2}$ in all figures.

DB of period $T_{b}=2.122$, place it in the rectilinear part of the chain, and follow its time evolution. During this evolution the DB energy alternates between the two extremes of having all the energy in the potential form and the other of having it completely in the kinetic form. We designate by $E_{D B}$ the internal breather energy and observe in Fig. 8 that it is to a high accuracy $\left(\sim 10^{-8}\right)$ constant. We note that in all DB energy estimations we calculate the energy by selecting a lattice window that includes all dominant DB lattice sites; the typical number of sites chosen is 11. According to Eqs. (3) and (5), and subsequent variable changes made in Secs. II and III A, the potential and kinetic energies are computed as follows:

$$
E_{p o t}=\sum_{n=n_{0}-5}^{n_{0}+5}\left\{\frac{1}{4} K_{1} a^{2}\left(\tau_{n}^{2}+\tau_{n+1}^{2}\right)+\frac{1}{8} \beta_{1} a^{4}\left(\tau_{n}^{4}+\tau_{n+1}^{4}\right)\right\}
$$

and

$$
E_{k i n}=\frac{1}{2} \sum_{n=n_{0}-5}^{n_{0}+5}\left\{\dot{x}_{n}^{2}+\dot{y}_{n}^{2}\right\}
$$

where $n_{0}$ is the central site of the DB, $\dot{x}_{n}$ and $\dot{y}_{n}$ are computed according to $\dot{x}_{n}=\dot{x}_{n-1}+a \dot{\tau}_{n} \cos \left(\theta_{n}\right)$, and $\dot{y}_{n}=\dot{y}_{n-1}$ $+a \dot{\tau}_{n} \sin \left(\theta_{n}\right)$, where $\left\{\theta_{n}\right\}$ are the initial fixed angles.

The total energy of the breather is thus

$$
E_{D B}=E_{p o t}+E_{k i n} .
$$

Since we do not have an exact Hamiltonian for the mFPU model for arbitrary nonuniform angles, i.e., in the bend region, we only use the exact expressions of the breather energy in the straight sections of the hairpin.

Let us now come to the case of a mobile breather; in order to investigate its dynamical profile we select appropriately a moving lattice window centered on the central DB site. The corresponding behavior for the breather in the rectilinear sec- 


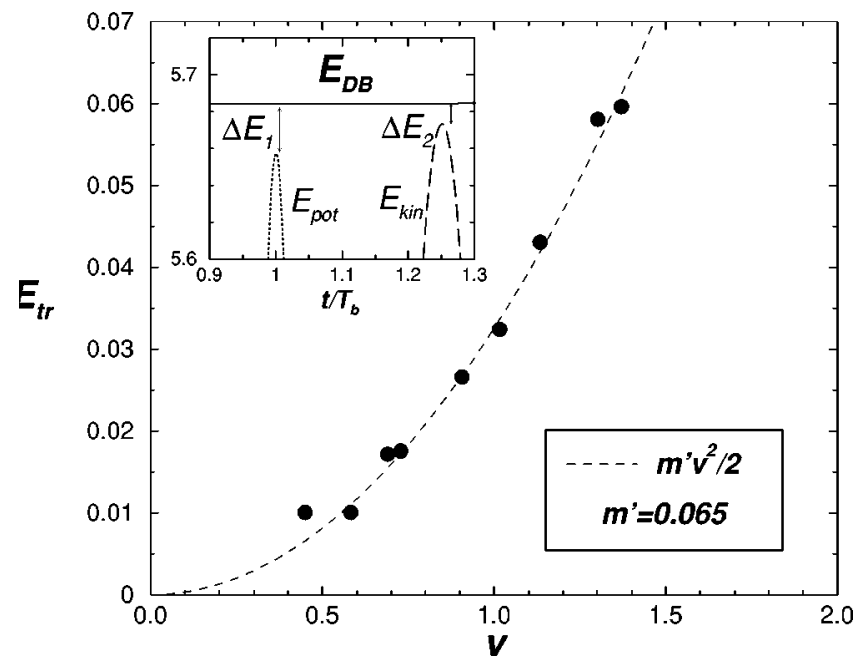

FIG. 9. Translational energy of a mobile DB in a straight line (FPU model) as a function of the velocity. The sound velocity here is 10 . The dashed line is a parabolic fit, corresponding to $v_{\min }=0$. Inset: Amplification of the energies of a mobile DB with $T_{b}$ $=2.122$ and $v_{0}=1.545$ in a straight line (FPU model). Velocities are in $0.1 a$ units.

tion of the hairpin is almost the same as in Fig. 8. The local energy exchanges between kinetic and potential energy seemingly proceed in a fashion similar to the one of the static DB. Nevertheless, some small in magnitude but clearly discernible differences emerge, as is seen in the inset of Fig. 9, which magnifies a selected segment. We can verify that neither the maximum potential nor the maximum kinetic energies are equal to $E_{D B}$ and additionally that there is a clear asymmetry between these two maximum values. The reason for this asymmetry clearly lies in the fact that the mobile DB should have some translational energy. Let us designate by $\Delta E_{1}, \Delta E_{2}$ the differences, respectively, of the maximum potential and kinetic breather energies from the total DB energy, $E_{D B}$. We would like to estimate the instantaneous translational breather energy. When $E_{k i n}=E_{k i n}^{\max }$ the difference $\Delta E_{2}$ is pure potential energy, while when $E_{\text {pot }}=E_{\text {pot }}^{\text {max }}$ the difference $\Delta E_{1}$ is pure kinetic DB energy. A good estimation thus for the translational DB energy is simply

$$
E_{t r}=E_{k i n}^{\max }-E_{\text {pot }}^{\max } \equiv \Delta E_{1}-\Delta E_{2}
$$

We observe in the inset of Fig. 9 that the translational DB energy $E_{\text {tras }}$ is only a small fraction of its total energy $E_{D B}$ ( $E_{\text {tras }}$ is at most $1 \%$ of $E_{D B}$ ). The evaluation of $E_{\text {tras }}$ is done after averaging over several DB periods $(\sim 50)$. The measured translational energy as a function of the DB velocity is plotted in Fig. 9. By fitting the translational energy as a kinetic energy of a compound object, we obtain an effective breather mass that turns out to be much smaller than the individual particle mass.

In order to explore the influence of the curved region in the total energy of the DB, we calculate the breather energy after crossing the curved region. Before entering into the curved region and after exiting, the DB energy decays exponentially with a very slow decay rate of order $\sim 10^{-6}$, due to the nonexact character of the mobile DB. There is no extra loss of energy just after exiting compared to just before entering. Therefore, we can consider that there are no appreciable energy losses as a result of motion in the curved part of the chain, and thus roughly speaking we can say that DB motion in curved chains conserves the breather energy.

As a result of this study of the restricted model we observe that the dynamics of DB's through a bent region of a polymer with angular rigidity can be parallelized to geometrical optics. Depending on their state, breathers are either reflected or refracted through the bent segment, which acts like a medium with an index of refraction different from that of the straight segment. In all cases, the energy of the localized packet is conserved, provided the DB is launched within a velocity window. In some sense, the curved, angularly rigid segment of the polymer acts as a gate or a filter that selects a breather depending on its individual characteristics, viz., its frequency and velocity.

\section{SECOND NEIGHBOR INTERACTIONS}

\section{A. Hairpin geometry}

In the preceding section, we investigated the dynamics of DB's in bend polymeric chains with only first neighbor interactions but with rigid angular constraints that enable one to fix the chain geometry to a desired shape. The angular constraint has been included so that the chain acquires rigidity and resembles more biomolecular systems rather than general homopolymers. Clearly, this angular restriction is very drastic, since it induces an artificially local strain in the chain, which, on the other hand, makes the analysis much easier. We will now lift this angular restriction in a way that enables the chain to retain rigidity around a prescribed shape. This will be done by including, in addition to first neighbor interactions, also second neighbor interactions. As noted in Sec. II, both first and second neighbor interactions will be taken to be similar but with different coefficients, i.e., symmetric quartic polynomials in the relative displacements between masses with different quadratic and quartic strengths. However, while the equilibrium spacing of the first neighbor interaction is taken to be the same constant across the chain, the next nearest neighbor distance $b_{n}$ varies locally in such a way that a desired geometry in the equilibrium chain structure is produced, as can be seen in Fig. 1(b). In particular, when the relative angle between two next neighboring masses is $\alpha_{n}$, their distance is chosen as $b_{n}=2 a \cos \left(\alpha_{n} / 2\right)$. The nearest neighbor nonlinear interaction is mostly responsible for the local longitudinal dynamics, whereas the next nearest neighbor interaction is for the geometric and angular rigidity of the chain. The next nearest neighbor interaction is thus playing a role similar to the angular constraint of the restricted model, while, on the other hand permitting transversally both flexibility and focusing.

Since we are interested in a comparison of the DB dynamics of the restricted model, we will impose the same hairpin geometry (see Fig. 3) on our polymeric chain and investigate several cases with different parameter values for the complete model introduced in Sec. II [Eqs. (8) and (9)]. Since interactions decay with distance, first neighbor interactions 


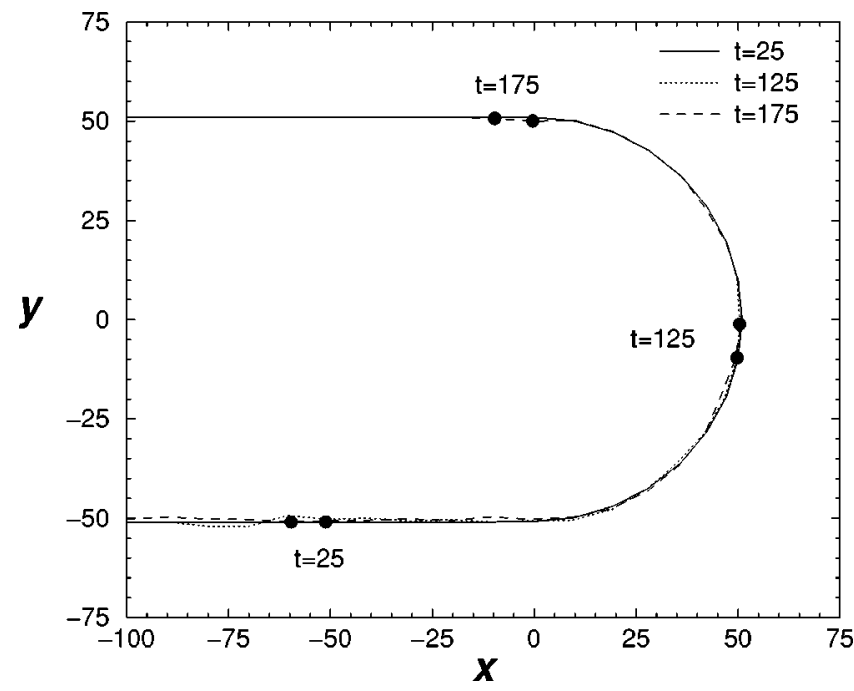

FIG. 10. Chain geometry and breather position at different times for a chain with first and second neighbor interactions $\left(K_{1}=\beta_{1}\right.$ $=1$ and $K_{2}=\beta_{2}=0.3$ ). The center, which occupies two sites, of the DB is marked with the black circles. The hairpin geometry is given by $\alpha=\pi / 16$ and $a=10 . x$ and $y$ are in units of $0.1 a$ in all figures.

are stronger than second neighbor interactions, i.e., $K_{1}>K_{2}$ and $\beta_{1}>\beta_{2}$. In this case, the almost exact longitudinal DB that is obtained from the algebraic method nearly coincides with the exact DB of the FPU model (no second neighbor interactions). We have worked with the same DB period, $T_{b}=2.122$, as before. We note that, by construction, our DB is accurate in the longitudinal direction of the chain but does not involve any transversal motion, and as a result it is not in general an exact breather for the arbitrarily shaped chain.

As in the preceding section, we are interested in the dynamics of a DB along a rigid curve. We first explore the effect of second neighbor interactions on the rigidity and stability of the chain. We consider a hairpin structure, launch the DB in the straight region, while removing any linear sound modes possibly induced initially on the lattice as a result of the breather kick. The propagation of the DB in a hairpin shaped chain with first and second interactions is shown in Fig. 10. We observe that the inclusion of second neighbor interactions adds rigidity and stability to the chain structure, the chain acts similarly to the rigidly constrained chain with only first neighbor interaction and DB motion being quite stable. We note that in the absence of both second neighbor interaction and angular constraints the chain dynamics is unstable yet DB propagation is still possible [26].

As regards the DB energy (Fig. 11), when the strength of the second neighbor interactions increase (the rigidity of the chain increases), the loss of energy when entering into the curved region is smaller, which is in agreement with the results of the restricted model. A small reduction of the DB frequency is also observed. Fine tuning of the second neighbor interaction values $K_{2}$ and $\beta_{2}$ can reduce these losses. The final velocity does not seem to depend very much on the harmonic or anharmonic nature of the second neighbor interactions nor on the strengths of the parameters. Note that the DB abruptly loses some energy while entering the curved

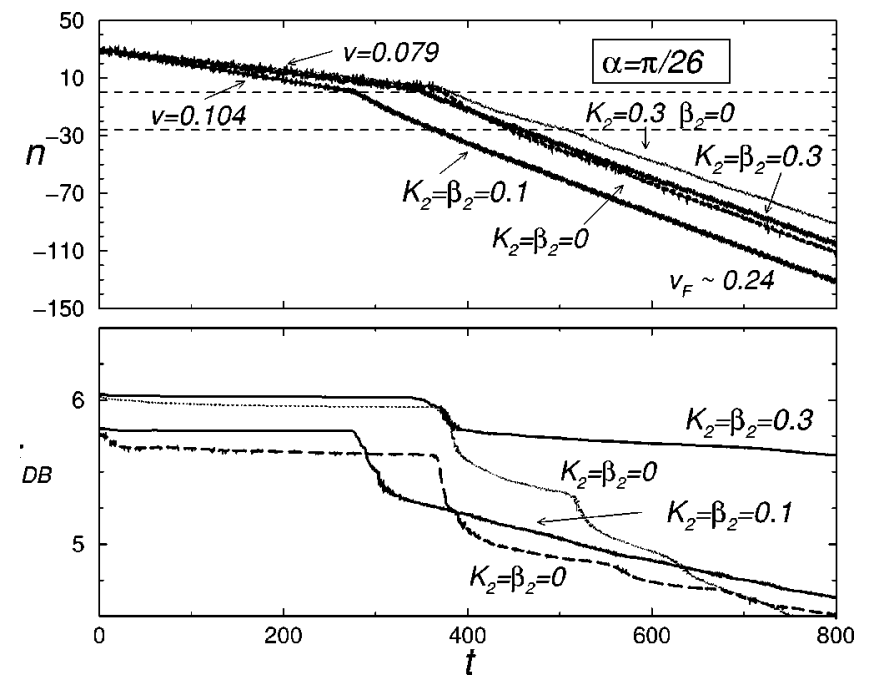

FIG. 11. Position of the DB center (top) and energy of the DB (bottom) as a function of time for a hairpin curvature given by $\alpha$ $=\pi / 26$ and $a=10$ as the second neighbor interactions are changed. Parameter values: $K_{1}=\beta_{1}=1$ and $T_{b}=2.122$.

region but not at the exit. This behavior will be analyzed at the end of the section.

As a result, with the inclusion of second neighbor interactions we obtain a rigid chain of masses that can sustain localized oscillations. In particular, the harmonic part of the second neighbor interaction, $K_{2}$, seems to be the main reason for the stability and rigidity of the chain, whereas the anharmonic part, $\beta_{2}$, increases the DB stability while reducing its energy loss. Other possible scenarios that mimic rigid chains are possible; see, for example, Ref. [28]. Now we proceed by studying the basic features of the DB kinetics on this chain with hairpin geometry.

As in the restricted model the DB enters the curved region for low curvatures and rebounces for high curvatures (see Fig. 12). However, since this model is not as rigid as the restricted one, the critical angle increases. Thus, the more rigid the chain is, the smaller the critical angle below which the DB rebounces seems to be and the less energy the DB loses when finding a curved region.

In contrast to the restricted model, the critical angle seems not to depend very much on the initial velocity of the DB, as seen in Fig. 12. In addition, we found that DB's always rebounce or exit the curved region with approximately the same asymptotic velocity $v_{F}$ independently of the initial velocity and the curvature. If the initial velocity of the DB is lower than $v_{F}$, the $\mathrm{DB}$ increases its velocity when entering into the curved region or while rebouncing (Fig. 12). If the initial velocity is above $v_{F}$, the $\mathrm{DB}$ decelerates when traversing the curved region (see Fig. 13). When it exits the curved part and enters in the second straight region, the change in velocity is much smaller than at the entrance. Thus, the hairpin is transparent for DB's with a velocity near $v_{F}$.

In this case the hairpin structure acts as a more active gate than in the restricted model. The curved segment can change the velocity of the DB, thus allowing it to transverse a possible next curve of a much more complicated biopolymer 

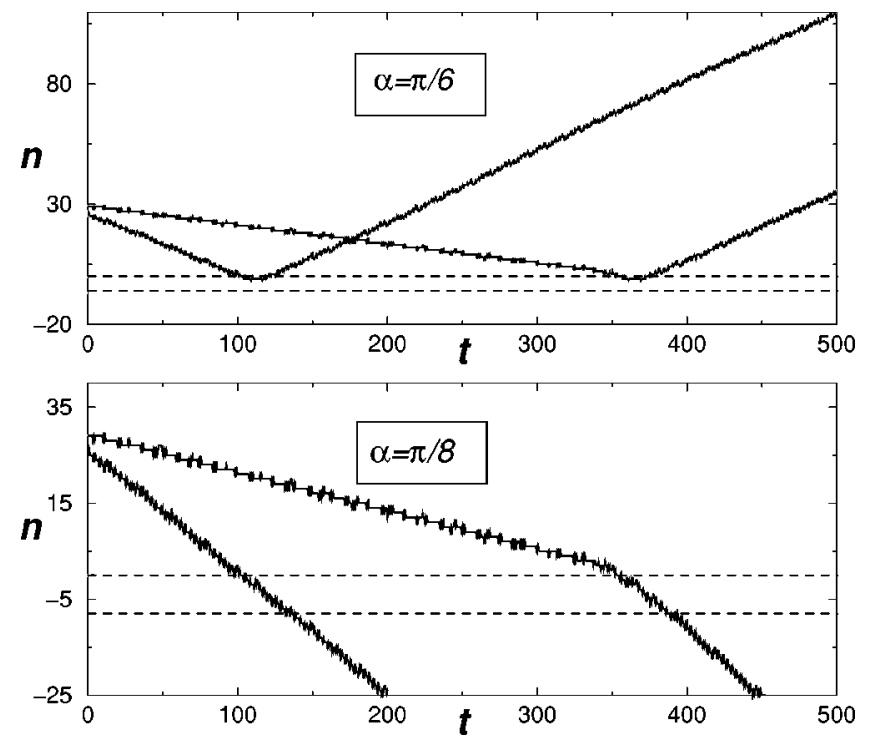

FIG. 12. Position of the DB center as a function of time for two initial velocities and two hairpin curvatures given by $\alpha=\pi / 6$ (top) and $\alpha=\pi / 8$ (bottom). Depending on the curvature, the DB enters into the curved region or rebounces. Parameter values: $K_{1}=\beta_{1}$ $=1, K_{2}=\beta_{2}=0.3$, and $T_{b}=2.122$.

without significant loss of energy and without significant change of speed.

\section{B. Alternative geometries}

The general features of DB motion that we presented for the polymer with first and second neighbor interactions are not particular to the hairpin geometry. In order to elucidate the influence of local or extended bends in the dynamics of breathers, we consider a sequence of lattice configurations with beginning and ending straight sections and a bend in between (Fig. 14). Performing the DB launching experiment in these chains we obtain the results that are depicted in Fig.

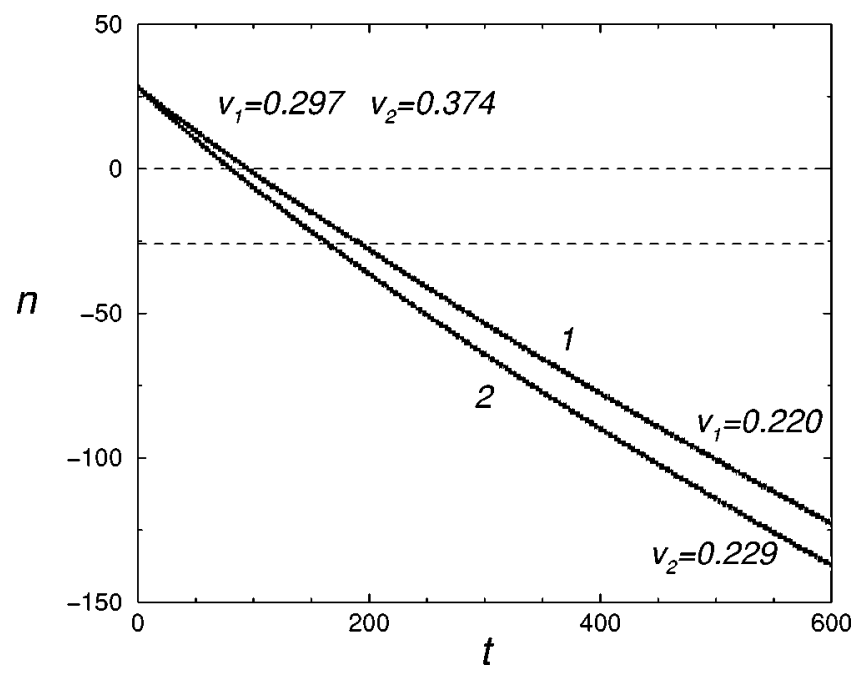

FIG. 13. Position of the DB center as a function of time for a hairpin curvature $\alpha=\pi / 26$ and different initial velocities. Same parameters as in the previous figure.

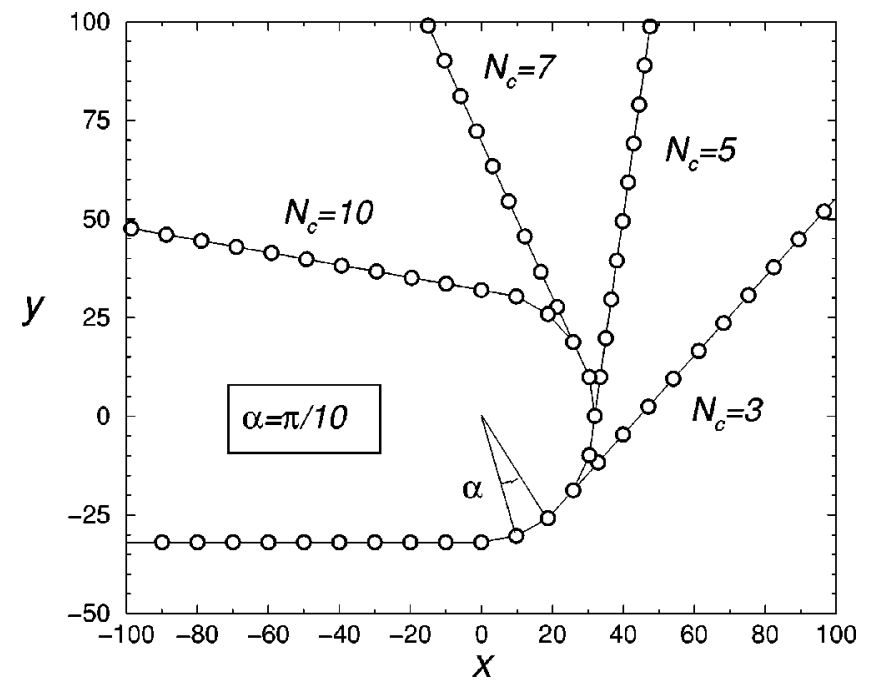

FIG. 14. Different chain geometries corresponding to two straight segments jointed by a curved region with the same curvature $\alpha=\pi / 10$. Distance between adjacent particles is $a=10$.

15 and can be summarized as follows: Upon interaction of the breather with the bend region there is a change in its motional state accompanied by a substantial loss of its energy. Subsequently, the breather accelerates to a velocity that changes very smoothly after exiting to reach an asymptotic velocity $v_{F}$ that does not depend on the length of the bend, and that coincides with the one previously found for the hairpin geometry. After the initial substantial drop of the breather energy, the rate of loss decreases and remains approximately constant for the duration of the simulation. We note that in all cases presented in Fig. 14, and in two of them plotted in Fig. 15, the terminal velocity $v_{F}$ is approximately constant, i.e., very weakly dependent on the specifics of the bend geometry.

As a result of this and the previous studies, we find that DB motion in the polymeric chains contains two seemingly

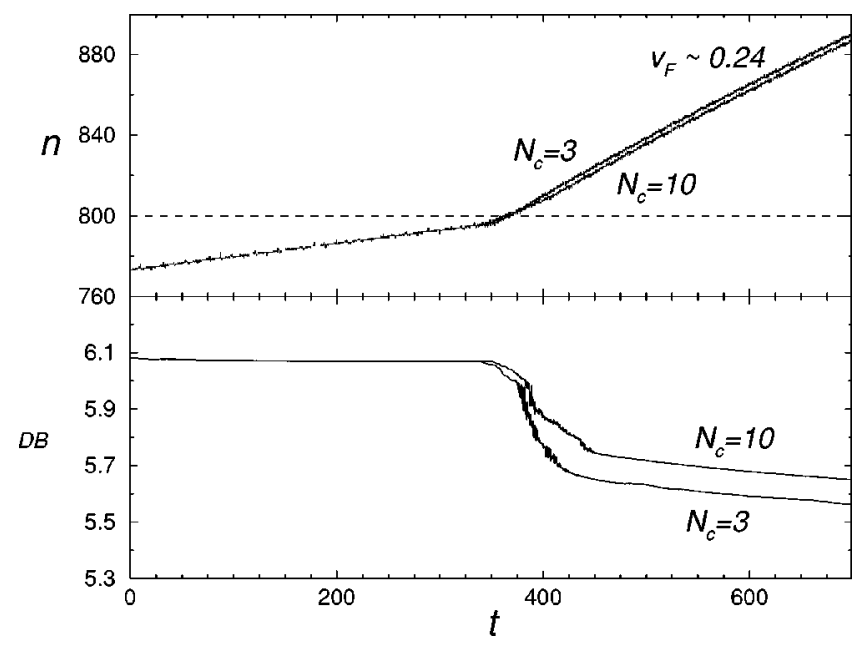

FIG. 15. (Top) Position of the DB center as a function of time for two of the geometries of the previous figure and (bottom) energies as a function of time. The parameter values are $K_{1}=\beta_{1}=1$, $K_{2}=\beta_{2}=0.3, a=10$, and the initial DB has period $T_{b}=2.122$. 


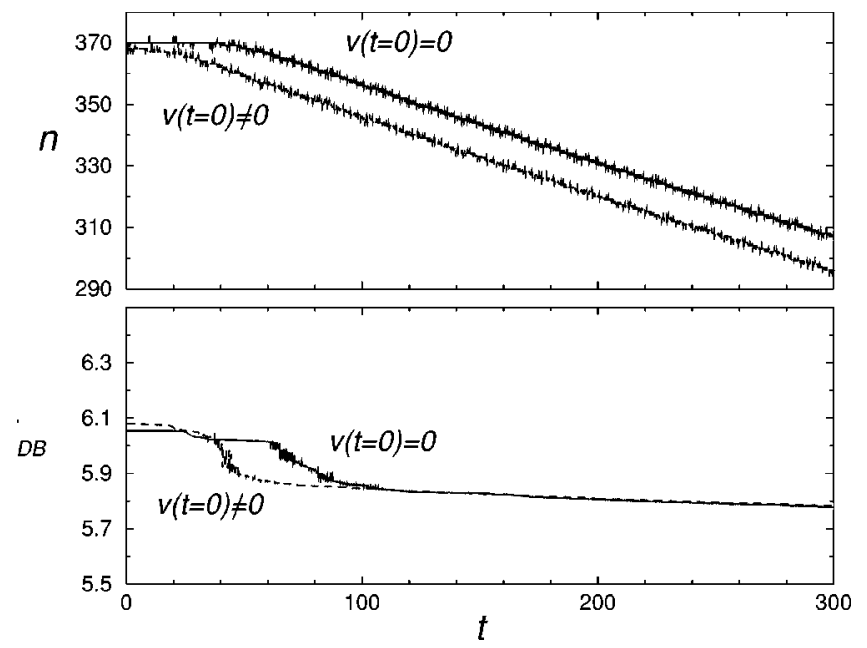

FIG. 16. (Top) Position of the DB center and (bottom) energies as a function of time for DB's in a straight chain. An initial static DB and a moving DB have been considered. In the former an initial perpendicular perturbation is made where the DB is set. In the latter, the $\mathrm{DB}$, while moving, finds the transverse perturbation. $\mathrm{Pa}-$ rameter values as in Fig. 15.

general features, i.e., that geometry induces an energy loss to the breather and also a selection mechanism for an optimalpropagational velocity. The energy loss is clearly induced initially at the bend region but it is preserved at much slower rates even when the breather returns in a rectilinear geometry. The terminal velocity, on the other hand, does not seem to be very sensitive to the specifics of the chain or the geometry of the bend and its value for the specific DB frequency studied is near $c / 4$, where $c$ is the speed of sound in the system. Since these phenomena are absent from both the general one-dimensional model as well as the restricted model with angular constraints, their source is related to the additional chain flexibility in the transversal direction as well as the fact that the initially injected breather is not an exact two-dimensional breather, either for the straight section or the bend region. Thus, we need to elucidate on the role that the transversal degrees of freedom play in the chain with first and second neighbor interactions; this will be done in the following section.

\section{Breather acceleration, damping through dephasing, and terminal velocity}

We consider now the polymeric chain with first and second neighbor interactions but include no geometric bend, i.e., we take it to be rectilinear. We set a breather in the usual way, adding, however, some small random perturbation in its initial transversal shape. Thus, the initially static longitudinal DB is found to oscillate on a two-dimensional chain. The results of this experiment are presented in Fig. 16. For an initially stationary breather $\left(v_{i}=0\right)$ with transversal perturbation we find that the breather initially loses some energy, begins moving, and shortly reaches the terminal velocity $v_{F}$, while at this stage its energy loss becomes substantially lower than the initial one. We thus find that an initial random perturbation in the transversal breather direction has effects

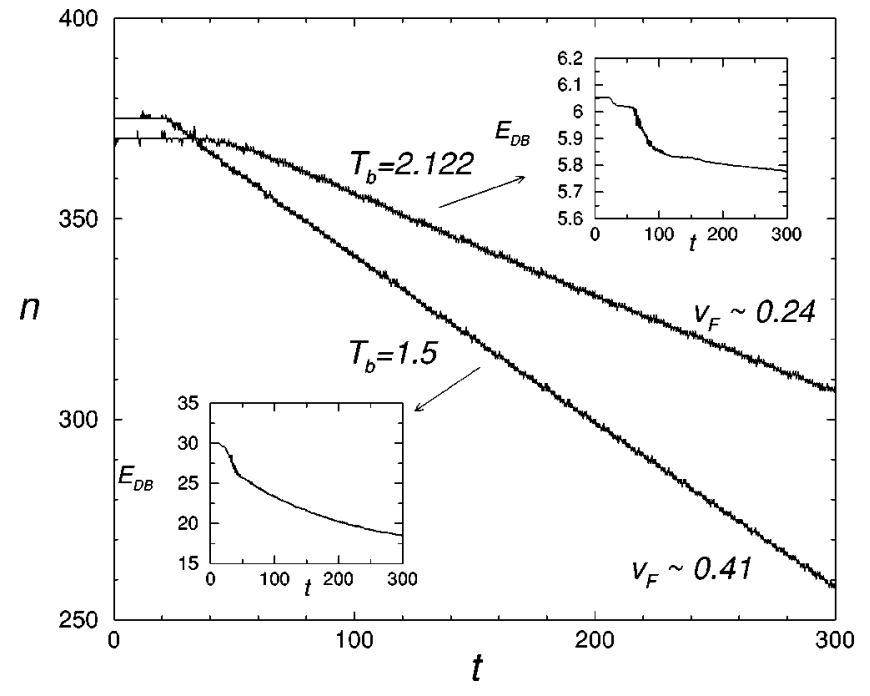

FIG. 17. Position of the DB center and energies as a function of time for two DB's with different period in a straight chain. The DB's are initially static and a transverse perturbation is made where the DB is located. Parameter values are as in Fig. 15.

very similar to those of the geometric bend. A similar result is observed if, for instance, the transversal perturbation is not included initially in the breather shape but is effected after it is launched at some other spatial region. We note that in all cases studied the small but not negligible breather energy loss persists at all times. As seen in Fig. 17 the asymptotic velocity $v_{F}$ and the rate of energy loss depend on the DB frequency. In Fig. 18 the explicit dependence of $v_{F}$ on the DB frequency $\omega_{b}$ is shown.

Finally, we stress that the existence of an asymptotic $v_{F}$ and a sudden decrease of the DB energy is not observed when longitudinal perturbations are set in the system, or when DB is crossed by phonons. Thus the features we have described are due to the flexibility of the chain, which can move in two dimensions.

We will now give qualitative arguments based on the accumulated numerical experimentation that points to the fact

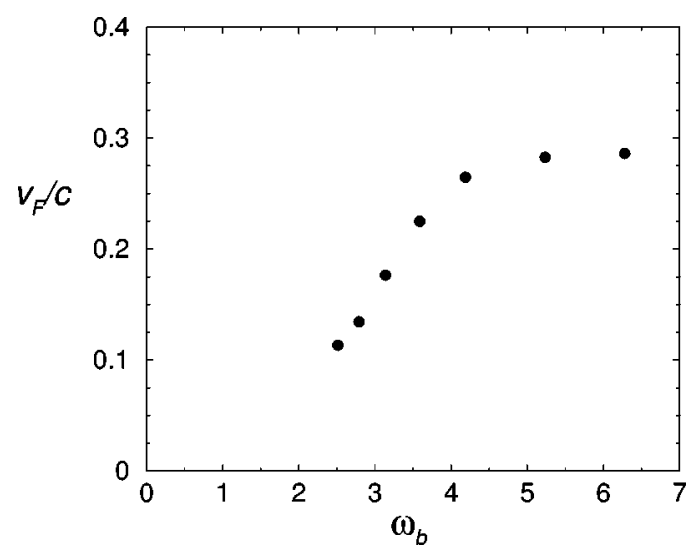

FIG. 18. Ratio between the asymptotic velocity and the sound velocity $\left(c=\sqrt{K_{1}+4 K_{2}}\right)$ of an initially static DB in a straight chain with second neighbor interactions and with a transverse perturbation applied on it vs DB frequency. The phonon band is below $\omega$ $\approx 2$. Parameter values are as in Fig. 15 . 
that the phenomena of breather acceleration (or deceleration), the onset of a terminal velocity, as well as the near constant energy loss stem from the same intricate nonlinear feedback mechanism that is operative between the coupled longitudinal and transversal polymer degrees of freedom. A quantitative explanation will be presented elsewhere. The feedback loop that leads to the observed facts involves the following links. Stage I: A coherent signal with frequency $\omega_{B}$ (breather) is injected longitudinally and either through perturbations or geometry (bend) couples to transversal degrees of freedom. Stage II: The horizontal-transversal coupling induces oscillatory motion in the center of mass of the particles in the transversal direction at a frequency $\omega_{T C M}$ that can be close but need not be identical to $\omega_{B}$. Stage III: Particles that move in the transversal direction with frequencies substantially different from $\omega_{T C M}$ introduce a dephasing mechanism that depends on the amount of their detuning as well as their amplitudes. This gives rise to damping through dephasing, a mechanism related to linear Landau damping [27] and that damps the transversal, and through the coupling to some extent, also the longitudinal oscillations. The dephasing mechanism now makes the coherent oscillation $\omega_{T C M}$ spectrally cleaner since it damps the more incoherent part of the transversal motion. Stage IV: The cleaner $\omega_{T C M}$ signal as well as the induced longitudinal oscillations in the tails couple resonantly through the interaction term to the main longitudinal one; since it is a higher order effect, in most cases it does not affect directly the coherent signal $\omega_{B}$, but couples to its modes depending on symmetry. If the transversal mode is antisymmetric (as in most cases presented here), it couples to the dominant DB antisymmetric mode, i.e., the pinning mode that is linearly responsible for DB motion [9] and accelerates the breather. As a result there is an increase in DB velocity until a characteristic maximal value $v_{F}$ that depends mainly on the DB frequency and not on the specific characteristics of the geometry and the interactions, as long as the chain has enough flexibility. In cases where the breather initially was injected with velocities higher than the terminal one, the dephasing induces damping through the coupling and can also lead to breather deceleration to the terminal velocity.

In Fig. 19 the longitudinal and transversal motions of neighboring sites of the chain are plotted for a hairpin geometry with $\alpha=\pi / 8$, when the change of speed and sudden decrease of energy occur. Note that while neighboring sites oscillate in the opposite phase in the longitudinal direction (thin lines), the phase of neighboring sites in the transversal motion changes from an opposite phase oscillation to an in phase oscillation. When this dephasing occurs, and thus the symmetry of oscillations in the transverse direction changes, the DB changes its speed and decreases its energy.

In defense of the previously presented mechanism, we note that in all cases studied numerically we typically observe DB energy loss that is approximately exponential and characterized by more than one exponent; the faster loss takes place when the signal encounters the transversal perturbation while the slower near constant loss occurs when the DB has reached the $v_{F}$ regime. Clearly, in the earlier stage there is substantially more dephasing leading to a larger and

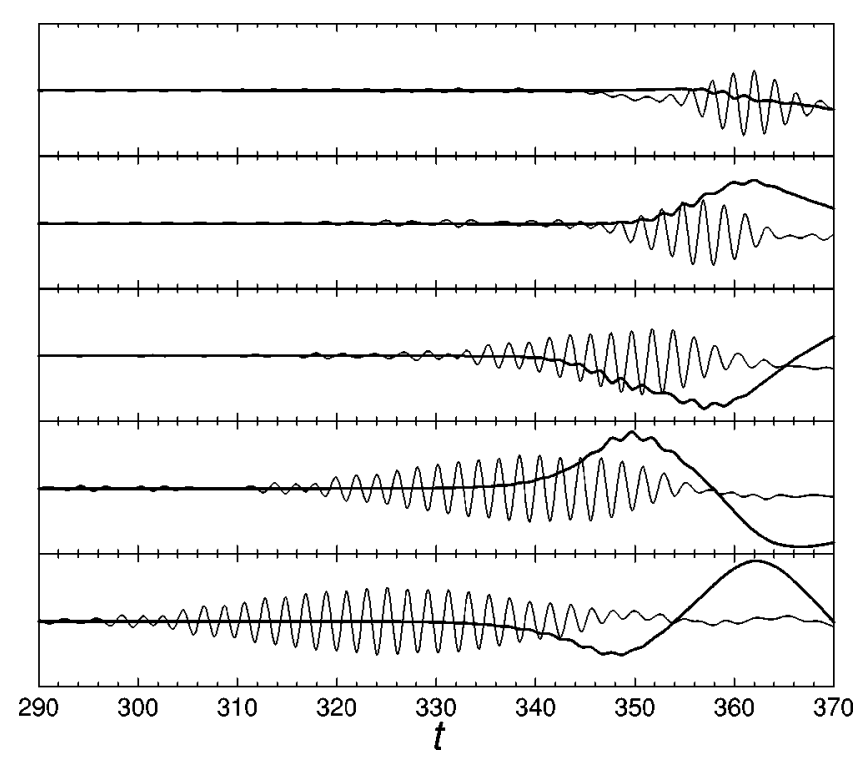

FIG. 19. Longitudinal (thin line) and tranverse (thick line) oscillations as a function of time, for different masses: $n, n+1$, $n+2, n+3$, and $n+4$ (from bottom to top). Parameter values are as in Fig. 15 and $\alpha=\pi / 8$.

faster energy loss. Additionally, when the induced transversal oscillation is not dominantly antisymmetric, no acceleration (or deceleration) to $v_{F}$ is observed, although damping through dephasing is clearly present [28]. Finally, since damping through dephasing is ubiquitous, there is a question as to whether this will lead asymptotically to DB destruction. Long term simulations have not shown either decay of the near constant damping rate or, however, substantial breather decay. It is possible, however, that after very long times, the linear dephasing regime ceases and nonlinearity comes back to rescue the breather from a linear death; this issue will have to be addressed through careful long term simulations.

\section{CONCLUSIONS}

Research work during the last decade has demonstrated that intrinsic localized modes are discrete lattice states with interesting dynamical and kinetic properties. Our main motivation in the present work has been to depart from simple lattice models and extend nonlinear localization research in more complex systems with additional properties such as elasticity. We chose as our basic model a simple polymerlike chain of masses connected via nonlinear springs that lies entirely on the plane. The main reason for selecting such a model has been our interest in extending breather research in the direction of biopolymers. In our polymeric nonlinear chain, we used short range first and second neighbor interactions only in order to understand their specific role in the dynamical processes without making the models too complex. Among the multiplicity of questions that one could ask in such a polymeric dynamical system, we focused primarily on the interplay of nonlinear localization with controlled polymer elasticity. Our methodology has been to use for the most part well-established, numerically accurate techniques for discrete breather construction, render them mobile, and 
subsequently study their transport properties in the system in various geometries. We used primarily the geometric configuration of a hairpin since it provides the typical shape in a $\beta$ sheet, one of the most abundant protein segment configurations.

In order to move the upward ladder of biomolecular complexity in a systematic and controllable fashion, we first studied a polymeric chain with only nearest neighbor interactions. Since the absence of other interactions makes the system too flexible to represent any biomolecule, we introduced rigidity by imposing angular constraints; as a result of the latter, our initial two-dimensional model with quartic interactions was transformed into a modified FPU model. This $\mathrm{mFPU}$ model is one dimensional and chain geometry is included in its coupling constants. The derivation of such an effective model was a major advance since it enabled us to investigate DB kinetics using standard one-dimensional techniques. In particular, we found that the DB's initially launched in a rectilinear segment of the hairpin can traverse the bend region by altering their velocity while in the bend and reverting back to their initial state after exiting this region. On other occasions, when the initial DB velocity is small, DB's cannot penetrate the bend region, but rebounce without appreciable energy loss. Thus the bend region acts as an active gate selecting a DB according to its frequency and velocity. By studying in detail the local energetics of the process we found that DB motion seems to be dictated by local energy conservation. During these processes the total DB energy is conserved to a high degree and, as a result, we do not observe substantial DB frequency shifts.

After establishing the basic properties of DB dynamics in the simple, constrained mFPU chain we moved on to a more complicated polymeric chain where second neighbor interactions were also included and were of the same form as those of the first neighbor ones. This system is more realistic and mimics better a biopolymer in that it uses the first neighbor interaction for its basic dynamics, the second neighbor one for structural stability, while ignoring longer-range interactions. The question of DB dynamics is now more complicated due to the possibility of affecting more extended chain segments, especially in bend regions. When an initially launched breather approaches the bend region, the presence of the second neighbor interaction extends the influence of the bend on the breather core before it enters the bend. In other words, the perturbation caused by the beginning of the bend region is felt by the breather center earlier than in the previous case and as result a more appreciable breather energy loss to the lattice is observed. The kinetic DB properties were studied extensively and we found two important differences in comparison to the mFPU constrained model: (a) There is substantial energy loss of the DB as it enters the curved region that persists at later times although at substantially reduced rates. The initial energy loss in the entry of the curve has a decay rate of order $10^{-3}$ or $10^{-4}$ while later the drop is of the order of $10^{-5}$. (b) As a result of the energy loss there is an appreciable change in the breather character and its kinetics. In particular, in most cases the DB accelerates to a higher velocity while in the bend region and retains its velocity upon exiting, in marked contrast to the behavior in the constrained chain. The basic reason for this behavior lies in the additional translational and rotational flexibility that the chain has now and the intricate feedback mechanism between the longitudinal and transversal degrees of freedom. This results in a more efficient DB energetic adaption to the local environment while the increase (or decrease) in speed is attributed to the resonant energy exchange between transverse and longitudinal internal modes of the breather and channeling of some additional energy into the translational degrees of freedom. The higher demand for strain energy in the bend is substantially offset by the second neighbor interactions, resulting in a DB with higher translational energy. The resulting state with a maximal velocity is seen to propagate efficiently also in the rectilinear segment since, due to the original energy loss, it is not possible energetically to revert to the originally injected state. In chains with stronger rigidity, where no such translational flexibility exists, like the mFPU chain presented here, or like the chain in Ref. [28], the existence of an asymptotic velocity was not observed.

As a result of this study the discrete breather emerges as an efficient energy transfer agent in more complex geometries. It is seen as able to be generated as a local depository of energy, transport it across chain segments with different local geometric properties, survive local environment changes, and adapt to the local strain requirements. Its dynamics does not seem to be lossless, nevertheless the magnitude of the energy loss is relatively small and most of it occurs at the interfaces with curvature changes. Even though its dynamics occurs with losses, it seems that it can cope with it by altering self-consistently its geometric configuration and dynamic state and thus its motion. The paradoxical behavior of an increase in DB velocity after a substantial energy loss is attributed to this effect and it critically depends on the presence of transversal degrees of freedom. We find thus that DB's can transfer localized energy quite efficiently in these chains and as a result we expect that they are possibly playing a crucial role in the bioenergetics of true biomolecules.

\section{ACKNOWLEDGMENTS}

This work has been supported partially by the European Union under the RTN project LOCNET (HPRN-CT-199900163) and by the Dirección General de Enseñanza Superior e Investigación Científica (Spain) under Project Nos. SAB1999-0059 and BFM2000-0624. M.I. also acknowledges the Ministerio de Educación Cultura y Deporte (Spain). This research was also partially supported by the U.S. Department of Energy under Contract No. W-7405ENG-36. 
[1] A.J. Sievers and S. Takeno, Phys. Rev. Lett. 61, 970 (1988).

[2] J.B. Page, Phys. Rev. B 41, 7835 (1990).

[3] S.R. Bickham, S.A. Kiselev, and A.J. Sievers, Phys. Rev. B 47, 14206 (1993).

[4] T. Dauxois and M. Peyrard, Phys. Rev. Lett. 70, 3935 (1993).

[5] S. Aubry, Physica D 71, 196 (1994).

[6] R.S. MacKay and S. Aubry, Nonlinearity 7, 1623 (1994).

[7] S. Aubry, Physica D 103, 201 (1996).

[8] J.L. Marin and S. Aubry, Nonlinearity 9, 1501 (1996).

[9] Ding Chen, S. Aubry, and G.P. Tsironis, Phys. Rev. Lett. 77, 4776 (1996).

[10] W.Z. Wang et al., Phys. Rev. Lett. 76, 3598 (1996).

[11] G.P. Tsironis and S. Aubry, Phys. Rev. Lett. 77, 5225 (1996); A. Bikaki, N.K. Voulgarakis, S. Aubry, and G.P. Tsironis, Phys. Rev. E 59, 1234 (1999).

[12] S. Flach and C.R. Willis, Phys. Rep. 295, 182 (1998).

[13] K.O. Rasmussen, S. Aubry, A.R. Bishop, and G.P. Tsironis, Eur. Phys. J. B 15, 169 (2000).

[14] G. Kopidakis and S. Aubry, Phys. Rev. Lett. 84, 3236 (2000).

[15] S. Aubry, G. Kopidakis, A.M. Morgante, and G.P. Tsironis, Physica B 296, 222 (2001); G. Kopidakis, S. Aubry, and G.P. Tsironis, Phys. Rev. Lett. 87, 165501 (2001).

[16] N.K. Voulgarakis, G. Kalosakas, A.R. Bishop, and G.P.
Tsironis, Phys. Rev. B 64, 020301 (2001).

[17] B.I. Swanson et al., Phys. Rev. Lett. 82, 3288 (1999).

[18] P. Binder et al., Phys. Rev. Lett. 84, 745 (2000).

[19] E. Trias, J.J. Mazo, and T.P. Orlando, Phys. Rev. Lett. 84, 741 (2000).

[20] A. Xie, L. VanderMeer, W. Hoff, and R.H. Austin, Phys. Rev. Lett. 84, 5435 (2000).

[21] Yu.B. Gaididei, S.F. Mingaleev, and P.L. Christiansen, Phys. Rev. E 62, R53 (2000).

[22] H. Frauenfelder, R. Austin, and P.G. Wolynes, Rev. Mod. Phys. 71(2), S419 (1999).

[23] G. P. Tsironis (unpublished).

[24] P.S. Lomdahl, O.H. Olsen, and M.R. Samuelsen, Phys. Lett. A 152, 343 (1991).

[25] A.V. Zolotaryuk, P.L. Christiansen, and A.V. Savin, Phys. Rev. E 54, 3881 (1996).

[26] G.P. Tsironis, M. Ibanẽs, and J.M. Sancho, Europhys. Lett. (to be published).

[27] A.W. Chao, Physics of Collective Beam Instabilities in High Energy Accelerators (Wiley, New York, 1993).

[28] R. Reigada, J.M. Sancho, M. Ibañes, and G.P. Tsironis, J. Phys. A 34, 8465 (2001). 